\title{
Load Analysis of Topology-Unaware TDMA MAC Policies for Ad-Hoc Networks
}

\author{
Konstantinos Oikonomou ${ }^{1}$ and Ioannis Stavrakakis ${ }^{2}$ \\ 1 INTRACOM S.A., Emerging Technologies \& Markets Department, \\ 19.5 Km Markopoulou Avenue, Paiania 190 02, Athens, Greece \\ Tel: +30 210 6677023, Fax: +30 2106671312 \\ okon@intracom.gr \\ 2 University of Athens, Department of Informatics \& Telecommunications \\ Panepistimiopolis, Ilissia 15 784, Athens, Greece \\ Tel: +30 2107275343 , Fax: +30 2107275333 \\ ioannis@di.uoa.gr
}

\begin{abstract}
Medium Access Control (MAC) policies in which the scheduling time slots are allocated irrespectively of the underline topology are suitable for ad-hoc networks, where nodes can enter, leave or move inside the network at any time. Topology-unaware MAC policies, that allocate slots deterministically or probabilistically have been proposed in the past and evaluated under heavy traffic assumptions. In this paper, the heavy traffic assumption is relaxed and the system throughput achieved by these policies is derived as a function of the traffic load. The presented analysis establishes the conditions and determines the values of the access probability for which the system throughput under the probabilistic policy is not only higher than that under the deterministic policy but it is also close to the maximum achievable, provided that the traffic load and the topology density of the network are known. In case the traffic load and/or the topology density are not known (which is commonly the case in ad-hoc networks), alternative values for the access probability are also derived which, although not the optimal (maximizing the system throughput), they do lead to a system throughput higher than that under the Deterministic Policy. Simulation results for a variety of topologies with different characteristics support the claims and the expectations of the analysis and show the comparative advantage of the Probabilistic Policy over the Deterministic Policy.
\end{abstract}

\section{Introduction}

The design of an efficient Medium Access Control (MAC) protocol in ad-hoc networks is challenging. The idiosyncrasies of these networks, where no infrastructure is present and nodes are free to enter, leave or move inside the network without prior configuration, allow for certain choices on the MAC design depending on the particular environment and therefore, it is not surprising that several MAC protocols have been proposed so far.

Contention-based MAC protocols are widely employed in ad-hoc networks, such as the CSMA/CA-based IEEE 802.11, [1]. In addition to the carrier sensing mechanism, MACA, [2], employs the Ready-To-Send/Clear-To-Send (RTS/CTS) handshake mechanism. This mechanism is mainly introduced to avoid the hidden/exposed terminal problem, which causes significant performance degradation in ad-hoc networks. Other protocols based on variations of the RTS/CTS mechanism have been proposed as well, [3], [4], [5].

TDMA-based MAC protocols are also employed, where each node is allowed to transmit during a specific set of TDMA scheduling time slots. S-TDMA - proposed by Kleinrock and Nelson, [6] - is capable of providing collision-free scheduling based on the exploitation of noninterfering transmissions in the network. In general, optimal solutions to the problem of time slot assignment often result in NP-hard problems, [7], [8], which are similar to the $n$-coloring problem in graph theory. Other collision-free scheduling schemes have been proposed as well, [9], [10], [11], [12].

Topology-unaware TDMA scheduling schemes determine the scheduling time slots irrespectively of the underlying topology and in particular, irrespectively of the scheduling time slots assigned to neighbor nodes. The topology-unaware scheme presented in [13], exploits the mathematical properties of polynomials with coefficients from finite Galois fields to randomly assign scheduling time slot sets to each node of the network. For each node it is guaranteed that at least one time slot in a frame would be collision-free, [13]. Another scheme, proposed in [14], maximizes the minimum guaranteed throughput. However, both schemes employ a deterministic policy (to be referred to as the Deterministic Policy) for the utilization of the assigned time slots that fails to utilize non-assigned time slots that could result in successful transmissions, [15], [16], [17]. Therefore, a new policy was proposed that probabilistically utilizes the non-assigned time slots according to a common access probability $p$ (to be referred to as the Probabilistic Policy). Analytical results for the maximization of the system throughput were derived and its dependency on the topology density was additionally investigated, [17]. An important feature of the Probabilistic Policy is that it is a simple transmission policy that can be implemented easily, allowing for transmissions without the need for coordination among different nodes or awareness about topological changes. 
All the aforementioned works ([13], [14], [15], [16], [17]) have focused on heavy traffic conditions and no work has been conducted for the non-heavy traffic case. In this paper, the probability $\lambda, 0 \leq \lambda \leq 1$, that a node has data for transmission during one time slot (probability $\lambda$ is also referred to as the traffic load) is considered. Analytical expressions for the system throughput are derived for both the Deterministic Policy and the Probabilistic Policy as a function of $\lambda$. An analysis based on approximations, whose accuracy is also investigated here, provides expressions for the system throughput maximization under the Probabilistic Policy, in case the traffic load and the topology density are known. In case the traffic load and/or the topology density are not known, expressions for the resulting throughput have also been derived; even though the system throughput under the Probabilistic Policy is not maximized, in this case, it turns out to be higher than that achieved under the Deterministic Policy. Extensive simulations confirm the results of the analysis.

In Section 2, an example ad-hoc network is described and some key definitions are introduced. Both transmission policies are described in Section 3. In Section 4, analytical expressions for the system throughput as a function of the traffic load are derived for both policies. In Section 5, the conditions for the existence of an efficient range of values for the access probability $p$ (values of the access probability $p$ under which the Probabilistic Policy outperforms the Deterministic Policy) are established through an approximate analysis. Furthermore, this analysis determines the value of the access probability that maximizes the system throughput provided that the traffic load and the topology density are known. In Section 6, the accuracy of the approximate analysis is studied, while in Section 7 the case when the traffic load and/or the topology density are not known is considered and expressions are derived such that the system throughput under the Probabilistic Policy, even though not maximized, is higher than that under the Deterministic Policy. At this point a comparison is carried out between the results of the analysis presented here for $\lambda=1$ and the results of the analysis presented in [17] (heavy traffic conditions). Simulation results, presented in Section 8 for a variety of topologies with different characteristics, support the claims and expectations addressed by the results of the analysis in the previous sections. Section 9 presents the conclusions.

\section{Network Definition}

An ad-hoc network may be viewed as a time varying multihop network and may be described in terms of a graph $G(V, E)$, where $V$ denotes the set of nodes and $E$ the set of links between the nodes at a given time instance. Let $|X|$ denote the number of elements in set $X$ and let $N=|V|$ denote the number of nodes in the network. Let $S_{u}$ denote the set of neighbors of node $u, u \in V$. These are the nodes $v$ to which a direct transmission from node $u$ (transmission $u \rightarrow v$ ) is possible. In this work omni-directional antennas will be considered and it is assumed that the transmission power is equal for all transmitting nodes in the network. Let $D$ denote the maximum number of neighbors for a node; clearly $\left|S_{u}\right| \leq D, \forall u \in V$. Time is divided into time slots with fixed duration and collisions with other transmissions is considered to be the only reason for a transmission not to be successful (corrupted).

Suppose that node $u$ wants to transmit to a particular neighbor node $v$ in a particular time slot $i$. In order for the transmission $u \rightarrow v$ to be successful (uncorrupted), two conditions should be satisfied. First, node $v$ should not transmit in the particular time slot $i$, or equivalently, no transmission $v \rightarrow \psi, \forall \psi \in S_{v}$ should take place in time slot $i$. Second, no neighbor of $v$ - except $u$ - should transmit in time slot $i$, or equivalently, no transmission $\zeta \rightarrow \chi, \forall \zeta \in S_{v}-\{u\}$ and $\chi \in S_{\zeta}$, should take place in time slot $i$. Consequently, transmission $u \rightarrow v$ is corrupted in time slot $i$ if at least one transmission $\chi \rightarrow \psi, \chi \in S_{v} \cup\{v\}-\{u\}$ and $\psi \in S_{\chi}$, takes place in time slot $i$. Figure 1 depicts an example network of 27 nodes. For this network $N=27$, and it can be seen that $D=6$. Nodes $\chi$, whose transmissions corrupt transmission $8 \rightarrow 13, \chi \in S_{13} \cup\{13\}-\{8\}$, are gray colored.

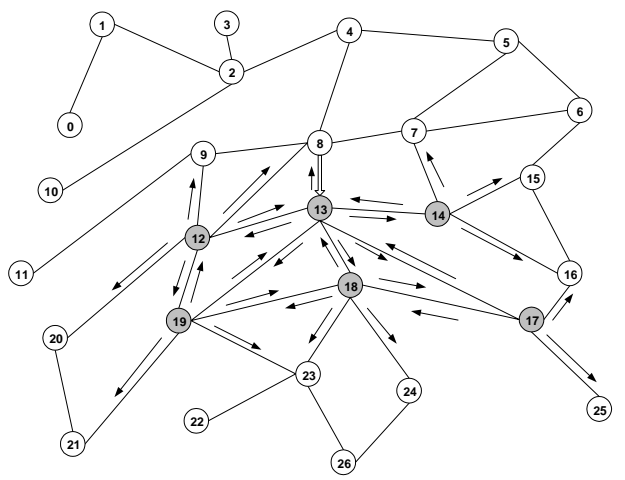

Fig. 1. Example network of 27 nodes for which $N=27$ and $D=6$. Nodes $\chi$ that their transmissions corrupt transmission $8 \rightarrow 13$, $\chi \in S_{13} \cup\{13\}-\{8\}=\{12,13,14,17,18,19\}$, are drawn gray. Transmission $8 \rightarrow 13$ is depicted by a white arrow, while those transmissions that corrupt transmission $8 \rightarrow 13$ are depicted by black arrows. 
In the event of a transmission $u \rightarrow v$, it is important for node $u$ to be notified about its successful (or corrupted) reception in order to retransmit the corresponding data in the case of corruption, [18]. This may be achieved by the use of retransmission mechanisms like the Selective Repeat ARQ mechanism. Another approach is to allow part of the end of each time slot to be used for the transmission of a positive acknowledgement (ACK) by the receiver (immediately after a successful transmission), [19]. Given that omni-directional antennas are used and the transmission power is equal for all transmitting nodes, the transmission corresponding to the positive acknowledgement ACK will not be corrupted by any other transmission. For example, suppose that transmission $8 \rightarrow 13$, for the network depicted in Figure 1, takes place in time slot $i$. If transmission $8 \rightarrow 13$ is successful (nodes 12,13,14,17, 18, 19 do not transmit), node 13 will transmit a positive acknowledgement ACK to node 8 at the end of time slot $i$. Transmission $13 \rightarrow 8$ may be corrupted if any of the nodes $12,9,4,7$ transmits at the same time. Given that in time slot $i$ transmission $8 \rightarrow 13$ was successful, it is obvious that none of the aforementioned nodes was the receiver node of a successful transmission (corruption would have taken place due to the presence of transmission $8 \rightarrow 13$ ). Consequently, these nodes will not transmit simultaneously with transmission $13 \rightarrow 8$ (at the end of time slot $i$ ) that acknowledges the successful transmission $8 \rightarrow 13$ in time slot $i$. For the rest it will be assumed that the acknowledgment is received instantaneously, [20].

\section{Transmission Policies}

According to the transmission policy proposed in [13] and [14], each node $u \in V$ is randomly assigned a unique polynomial $f_{u}$ of degree $k$ with coefficients from a finite Galois field of order $q(G F(q))$. Polynomial $f_{u}$ is represented as $f_{u}(x)=$ $\sum_{i=0}^{k} a_{i} x^{i}(\bmod q),[14]$, where $a_{i} \in\{0,1,2, \ldots, q-1\}$; parameters $q$ and $k$ are calculated based on $N$ and $D$, according to the algorithm presented either in [13] or [14].

The access scheme considered is a TDMA scheme with a frame consisted of $q^{2}$ time slots. If the frame is divided into $q$ subframes $s$ of size $q$, then the time slot assigned to node $u$ in subframe $s,(s=0,1, \ldots, q-1)$ is given by $f_{u}(s)$ mod $q$, [14]. Consequently, one time slot is assigned for each node in each subframe. Let $\Omega_{u}$ be the the set of time slots assigned to node $u$. Given that the number of subframes is $q,\left|\Omega_{u}\right|=q$.

The deterministic transmission policy, proposed in [13] and [14], is the following.

The Deterministic Policy: Each node $u$ transmits in a slot $i$ only if $i \in \Omega_{u}$, provided that it has data to transmit.

The relation between $N, D, q$ and $k$ is important in order to explain the main property of the Deterministic Policy: there exists at least one time slot in a frame over which a specific transmission will remain uncorrupted, [13]. Suppose that two neighbor nodes $u$ and $v$ have been assigned two (unique) polynomials $f_{u}$ and $f_{v}$ of degree $k$, respectively. Given that the roots of each node's polynomial correspond to the assigned time slots to each node, $k$ common time slots is possible to be assigned among two neighbor nodes. Given that $D$ is the maximum number of neighbor nodes of any node, $k D$ is the maximum number of time slots over which a transmission from any node is possible to become corrupted. Since the number of time slots that a node is allowed to transmit in a frame is $q$, if $q>k D$ or $q \geq k D+1(k$ and $D$ are integers) is satisfied, there will be at least one time slot in a frame in which a specific transmission will remain uncorrupted for any node in the network, [13].

The assigned unique polynomials $f_{u}$ to each node $u$ allow for the determination of $\Omega_{u}$. This assignment may be considered as similar to MAC identification numbers (MAC IDs) and are assigned to each node accordingly: either they are included in the device or they are assigned using a control mechanism. In this work, it is assumed a node has been assigned a unique polynomial randomly (without taking into account the neighbor nodes of the node and/or their assigned polynomials) before the node enters the network. It is also important to guarantee that the number of unique polynomials is enough for all nodes in the network, or $q^{k+1} \geq N$. If for a given $N$ and $q, q^{k+1} \geq N$ is not satisfied, then $k$ has to be increased (perhaps resulting to a new $q$ that satisfies $q>k D$ ) until the number of unique polynomials is sufficient.

Let $O_{i, u \rightarrow v}$ be that set of nodes $\chi$ whose transmissions corrupt a particular transmission $u \rightarrow v\left(\right.$ i.e. $\chi \in S_{v} \cup\{v\}-\{u\}$ ) and which are also allowed to transmit in time slots $i$ (i.e., they are assigned slot $i$ or $i \in \Omega_{\chi}$ ). Let $O_{i, u \rightarrow v}{ }^{\mathfrak{c}}$ be the complementary set of nodes in $S_{v} \cup\{v\}-\{u\}$ for which $i \notin \Omega_{\chi}$.

$$
\begin{aligned}
O_{i, u \rightarrow v} & =\left\{\chi: \chi \in S_{v} \cup\{v\}-\{u\}, i \in \Omega_{\chi}\right\}, \\
O_{i, u \rightarrow v}{ }^{\mathfrak{c}} & =\left\{\chi: \chi \in S_{v} \cup\{v\}-\{u\}, i \notin \Omega_{\chi}\right\} .
\end{aligned}
$$

Obviously, $\left|O_{i, u \rightarrow v}\right|+\left|O_{i, u \rightarrow v}{ }^{\mathfrak{c}}\right|=\left|S_{v}\right|$.

Depending on the particular random assignment of the polynomials, it is possible that two nodes be assigned overlapping time slots (i.e., $\Omega_{u} \cap \Omega_{v} \neq \emptyset$ ). Let $C_{u \rightarrow v}$ be the set of overlapping time slots between those assigned to node $u$ 
and those assigned to any node $\chi \in S_{v} \cup\{v\}-\{u\}$.

$$
C_{u \rightarrow v}=\Omega_{u} \cap\left(\bigcup_{\chi \in S_{v} \cup\{v\}-\{u\}} \Omega_{\chi}\right) .
$$

Obviously, if $i \in C_{u \rightarrow v}\left(\left|O_{i, u \rightarrow v}\right|>0\right.$ when $\left.i \in C_{u \rightarrow v}\right)$, it is possible that another transmission will corrupt transmission $u \rightarrow v$ in time slot $i$, provided that there are data for transmission. If $i \in \Omega_{u}-C_{u \rightarrow v}\left(\left|O_{i, u \rightarrow v}\right|=0\right)$, no transmission corrupts transmission $u \rightarrow v$ in time slot $i$.

Let $R_{u \rightarrow v}$ denote the set of time slots $i, i \notin \Omega_{u}$, over which transmission $u \rightarrow v$ would be successful even for heavy traffic conditions $(\lambda=1)$. Equivalently, $R_{u \rightarrow v}$ contains those slots not included in set $\bigcup_{\chi \in S_{v} \cup\{v\}} \Omega_{\chi}$. Consequently,

$$
\left|R_{u \rightarrow v}\right|=q^{2}-\left|\bigcup_{\chi \in S_{v} \cup\{v\}} \Omega_{\chi}\right| .
$$

As it has been shown in [17], $\left|R_{u \rightarrow v}\right| \geq q(k-1) D$, resulting in a large number of unused time slots (especially for $k>1$ ) that could be used to increase the average number of transmissions. In general, the use of slots $i, i \in R_{u \rightarrow v}$, may increase the average number of successful transmissions, as long as $R_{u \rightarrow v}$ is determined and time slots $i \in R_{u \rightarrow v}$ are used efficiently.

The determination of $R_{u \rightarrow v}$ requires the existence of a mechanism for the extraction of sets $\Omega_{\chi}, \forall \chi \in S_{v}$. In addition, the efficient use of slots in $R_{u \rightarrow v}$ by node $u$, requires further coordination and control exchange with neighbor nodes $\chi$, whose transmissions $\chi \rightarrow \psi$, with $R_{\chi \rightarrow \psi} \cap R_{u \rightarrow v} \neq \emptyset$, may utilize the same slots in $R_{\chi \rightarrow \psi} \cap R_{u \rightarrow v}$ and corrupt either transmission $u \rightarrow v$ or $\chi \rightarrow \psi$, or both. In order to use all non-assigned time slots without the need for further coordination among the nodes, the following probabilistic transmission policy was introduced in [17].

The Probabilistic Policy: Each node $u$ always transmits in slot $i$ if $i \in \Omega_{u}$ and transmits with probability $p$ in slot $i$ if $i \notin \Omega_{u}$, provided it has data to transmit.

The Probabilistic Policy does not require specific topology information (e.g., knowledge of $R_{u \rightarrow v}$, etc.) and, thus, induces no additional control overhead. The access probability $p$ is a simple parameter common for all nodes. Under the Probabilistic Policy, all slots $i \notin \Omega_{u}$ are potentially utilized by node $u$ : both, those in $R_{u \rightarrow v}$, for a given transmission $u \rightarrow v$, as well as those not in $\Omega_{u} \cup R_{u \rightarrow v}$ that may be left unutilized by neighboring nodes under non-heavy traffic conditions $(\lambda<1)$.

Following the approach described in [13], and regarding the example network depicted in Figure 1, $q, k$ and each set $\Omega_{\chi}$ are determined for all nodes $\chi$ in the network. In particular, $q=7, k=1$ and for transmission $8 \rightarrow 13, C_{8 \rightarrow 13}$, $R_{8 \rightarrow 13}$, and $\Omega_{\chi}, \chi \in S_{13} \cup\{13\}$, are determined and depicted in Figure 2. Time slots $i \in \Omega_{8}-C_{8 \rightarrow 13}$ are depicted with light-gray colored boxes, while time slots $i \in C_{8 \rightarrow 13}$ are depicted with dark-gray colored boxes. Time slots $i \in R_{8 \rightarrow 13}$ are depicted with white colored boxes. Finally, time slots $i \notin \Omega_{8} \cup R_{8 \rightarrow 13}$ are depicted with mid-gray colored boxes. The upper left small numbers in the boxes refer to the particular time slot in a frame, while the central number refer to the nodes that have been assigned the particular time slot.

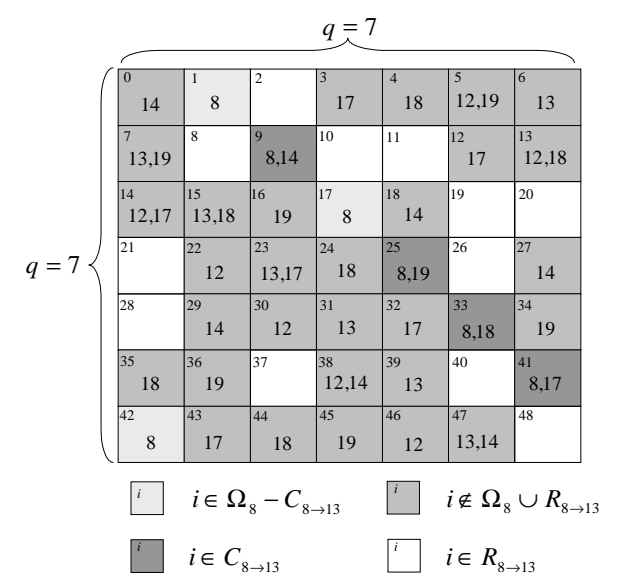

Fig. 2. Transmission sets $\Omega_{\chi}, \chi \in S_{13} \cup\{13\}=\{8,12,13,14,17,18,19\} . \Omega_{8}=\{1,9,17,25,33,41,42\}, C_{8 \rightarrow 13}=\{9,25,33,41\}$ $\left(\left|C_{8 \rightarrow 13}\right|=4\right)$ and $R_{8 \rightarrow 13}=\{2,8,10,11,19,20,21,26,28,37,40,48\}\left(\left|R_{8 \rightarrow 13}\right|=12\right)$. 


\section{System Throughput}

In order to derive the expressions for the system throughput under both the Deterministic Policy and the Probabilistic Policy, it is necessary to derive the expressions for the probability of success of a specific transmission (throughput) under both policies.

\subsection{Specific Transmission}

Let $P_{i, D, u \rightarrow v}\left(P_{i, P, u \rightarrow v}\right)$ denote the probability that transmission $u \rightarrow v$ in slot $i$ is successful, under the Deterministic (Probabilistic) Policy. Let $P_{D, u \rightarrow v}\left(P_{P, u \rightarrow v}\right)$ be the average probability over a frame for transmission $u \rightarrow v$ to be successful during a time slot, under the Deterministic (Probabilistic) Policy. That is, $P_{D, u \rightarrow v}=\frac{1}{q^{2}} \sum_{i=1}^{q^{2}} P_{i, D, u \rightarrow v}$ $\left(P_{P, u \rightarrow v}=\frac{1}{q^{2}} \sum_{i=1}^{q^{2}} P_{i, P, u \rightarrow v}\right)$, where $q^{2}$ is the frame size, in time slots.

Under the Deterministic Policy each node $u$ transmits over a time slot $i$ with probability $\lambda$, if $i \in \Omega_{u}$. Any other node $\chi$, for which $i \in \Omega_{\chi}$, also transmits with probability $\lambda$. Consequently, $P_{i, D, u \rightarrow v}=0$, for $i \notin \Omega_{u}$, and $P_{i, D, u \rightarrow v}=$ $\lambda(1-\lambda)^{\left|O_{i, u \rightarrow v}\right|}$, for $i \in \Omega_{u}$. Given that $\left|O_{i, u \rightarrow v}\right|=0$ for those slots $i \in \Omega_{u}-C_{u \rightarrow v}\left(\left|O_{i, u \rightarrow v}\right|>0\right.$ when $\left.i \in C_{u \rightarrow v}\right)$, it is concluded that $P_{i, D, u \rightarrow v}=\lambda$, for $i \in \Omega_{u}-C_{u \rightarrow v}$. As a result,

$$
P_{D, u \rightarrow v}=\frac{q-\left|C_{u \rightarrow v}\right|+\sum_{i \in C_{u \rightarrow v}}(1-\lambda)^{\left|O_{i, u \rightarrow v}\right|}}{q^{2}} \lambda,
$$

where $\left|\Omega_{u}\right|=q$.

Under the Probabilistic Policy, if $i \in \Omega_{u}$, each node $u$ transmits over a time slot $i$ with probability $\lambda$, while if $i \notin \Omega_{u}$ each node $u$ transmits with probability $p \lambda$. Any other node $\chi$, for which $i \in \Omega_{\chi}$, transmits in the same time slot $i$ with probability $\lambda$, whereas if $i \notin \Omega_{\chi}$ it transmits with probability $p \lambda$. Consequently, for $i \in \Omega_{u}, P_{i, P, u \rightarrow v}=\lambda(1-\lambda)^{\left|O_{i, u \rightarrow v}\right|}(1-$ $p \lambda)^{\left|O_{i, u \rightarrow v}{ }^{\mathfrak{c}}\right|}$, while for $i \notin \Omega_{u}, P_{i, P, u \rightarrow v}=p \lambda(1-\lambda)^{\left|O_{i, u \rightarrow v}\right|}(1-p \lambda)^{\left|O_{i, u \rightarrow v}{ }^{\mathfrak{c}}\right|}$. Given that $\left|O_{i, u \rightarrow v}\right|+\left|O_{i, u \rightarrow v}{ }^{\mathfrak{c}}\right|=\left|S_{v}\right|$, for $i \in \Omega_{u}, P_{i, P, u \rightarrow v}=\lambda\left(\frac{1-\lambda}{1-p \lambda}\right)^{\left|O_{i, u \rightarrow v}\right|}(1-p \lambda)^{\left|S_{v}\right|}$, while for $i \notin \Omega_{u}, P_{i, P, u \rightarrow v}=p \lambda\left(\frac{1-\lambda}{1-p \lambda}\right)^{\left|O_{i, u \rightarrow v}\right|}(1-p \lambda)^{\left|S_{v}\right|}$. By definition, $\left|O_{i, u \rightarrow v}\right|=0$, if $i \in\left(\Omega_{u}-C_{u \rightarrow v}\right) \cup R_{u \rightarrow v}$, and $\left|O_{i, u \rightarrow v}\right|>0$ if $i \in C_{u \rightarrow v}$ or $i \notin \Omega_{u} \cup R_{u \rightarrow v}$. As a result,

$$
\begin{aligned}
P_{P, u \rightarrow v}= & \frac{\sum_{i \in C_{u \rightarrow v}}\left(\frac{1-\lambda}{1-p \lambda}\right)^{\left|O_{i, u \rightarrow v}\right|}}{q^{2}} \lambda(1-p \lambda)^{\left|S_{v}\right|} \\
& +\frac{q-\left|C_{u \rightarrow v}\right|+p\left|R_{u \rightarrow v}\right|}{q^{2}} \lambda(1-p \lambda)^{\left|S_{v}\right|} \\
& +\frac{p \sum_{i \notin R_{u \rightarrow v} \cup \Omega_{u}}\left(\frac{1-\lambda}{1-p \lambda}\right)^{\left|O_{i, u \rightarrow v}\right|}}{q^{2}} \lambda(1-p \lambda)^{\left|S_{v}\right|} .
\end{aligned}
$$

\subsection{System Throughout}

The destination node $v$ of a particular transmission $u \rightarrow v$, depends on the destination of the data and consequently, on the network application as well as on the routing protocol. For the rest of this work it will be assumed that a node $u$ transmits to only one $v, v \in S_{u}$. For any of the numerical or simulation results presented in the sequel, node $v$ will be a node randomly selected out of $S_{u}$.

The probability of success of a transmission (averaged over all nodes in the network) under each corresponding policy, is referred to as the system throughput and is denoted by $P_{D}\left(P_{P}\right)$ under the Deterministic (Probabilistic) Policy. According to equations (5) and (6), the system throughput $P_{D}$ and $P_{P}$ is derived from the following equations.

$$
\begin{aligned}
P_{D}= & \frac{1}{N} \sum_{\forall u \in V} \frac{q-\left|C_{u \rightarrow v}\right|+\sum_{i \in C_{u \rightarrow v}}(1-\lambda)^{\left|O_{i, u \rightarrow v}\right|}}{q^{2}} \lambda, \\
P_{P}= & \frac{1}{N} \sum_{\forall u \in V} \frac{\sum_{i \in C_{u \rightarrow v}}\left(\frac{1-\lambda}{1-p \lambda}\right)^{\left|O_{i, u \rightarrow v}\right|}}{q^{2}} \lambda(1-p \lambda)^{\left|S_{v}\right|} \\
& +\frac{1}{N} \sum_{\forall u \in V} \frac{q-\left|C_{u \rightarrow v}\right|+p\left|R_{u \rightarrow v}\right|}{q^{2}} \lambda(1-p \lambda)^{\left|S_{v}\right|}
\end{aligned}
$$




$$
+\frac{1}{N} \sum_{\forall u \in V} \frac{p \sum_{i \notin R_{u \rightarrow v} \cup \Omega_{u}}\left(\frac{1-\lambda}{1-p \lambda}\right)^{\left|O_{i, u \rightarrow v}\right|}}{q^{2}} \lambda(1-p \lambda)^{\left|S_{v}\right|},
$$

where $v \in S_{u}$.

From Equation (7) and Equation (8) it can be seen that for $p=0, P_{P}=P_{D}$, while for $p=1, P_{P}=\frac{1}{N} \sum_{\forall u \in V} \lambda(1-$ $\lambda)^{\left|S_{v}\right|}$. The latter is greater than zero for $0<\lambda<1$.

In Figure 3(a) the system throughput for the network depicted in Figure 1 is illustrated for various values of $p$, as a function of $\lambda$. It is obvious that as $\lambda$ increases, $P_{P}$ increases faster than $P_{D}$ until a maximum is assumed. If the maximum is assumed for $\lambda<1$, then as $\lambda$ increases further, $P_{P}$ decreases until $\lambda=1$. As it can also be observed, those values of $p$ for which $P_{P}$ is large for small values of $\lambda$, result in small $P_{P}$ for large values of $\lambda$ and vice versa. Figure 3(b) depicts $P_{P}$ as a function of $p$ for different values of $\lambda$; the same conclusion as before may be drawn.

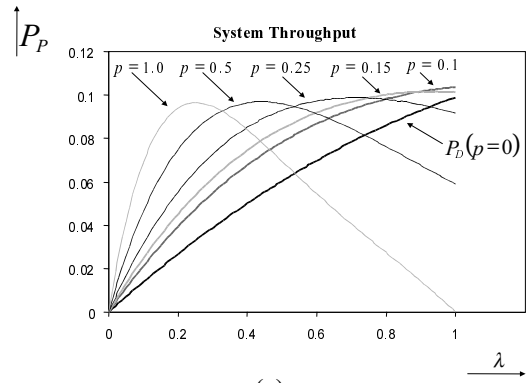

(a)

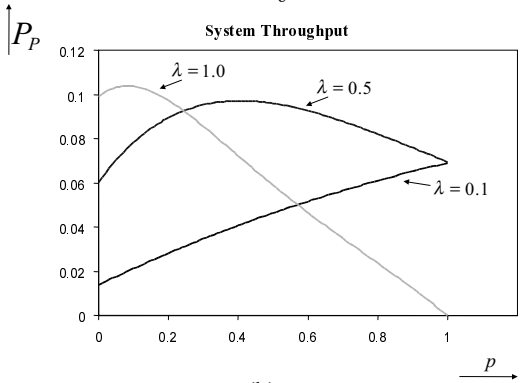

(b)

Fig. 3. Numerical results for the system throughput, $P_{P}$, under the Probabilistic Policy (a) as a function of $\lambda$, for different values of $p(p=0, p=0.1, p=0.15, p=0.25, p=0.5$ and $p=1.0)$ and (b) as a function of $p$, for different values of $\lambda(\lambda=0.1, \lambda=0.5$ and $\lambda=1.0)$. Note that for $p=0, P_{P}=P_{D}$.

From the above discussion it is obvious that a certain value for $p$ maximizes the system throughput for a certain value of $\lambda$. The aim of the following section is to establish the conditions under which $P_{P} \geq P_{D}$ is satisfied and derive the relations between $\lambda$ and $p$ for which not only $P_{P} \geq P_{D}$, but $P_{P}$ is also maximized.

\section{System Throughput Maximization}

As it is difficult to derive analytical expressions and establish the conditions for the system throughput maximization from Equation (8), a more tractable form of $P_{P}$ is considered.

Let $\hat{P}_{P}$ denote that value of $P_{P}$ when $\frac{1-\lambda}{1-p \lambda}$ is replaced by 1 . Then, $\hat{P}_{P}=\frac{1}{N} \sum_{\forall u \in V} \frac{\left|C_{u \rightarrow v}\right|}{q^{2}} \lambda(1-p \lambda)^{\left|S_{v}\right|}+\frac{1}{N} \sum_{\forall u \in V} \frac{q-\left|C_{u \rightarrow v}\right|+p \mid R_{u}}{q^{2}}$ $p \lambda)^{\left|S_{v}\right|}+\frac{1}{N} \sum_{\forall u \in V} \frac{p\left(q^{2}-q-\left|R_{u \rightarrow v}\right|\right)}{q^{2}} \lambda(1-p \lambda)^{\left|S_{v}\right|}$ or, finally,

$$
\hat{P}_{P}=\frac{1}{N} \sum_{\forall u \in V} \frac{1+p(q-1)}{q} \lambda(1-p \lambda)^{\left|S_{v}\right|} .
$$

Since $\frac{1-\lambda}{1-p \lambda} \leq 1$, it is clear that $\hat{P}_{P} \geq P_{P}$.

Even though $\hat{P}_{P}$ has a more tractable form than that of $P_{P}$, it still cannot be easily analyzed further. It can be seen from Equation (9) that $\hat{P}_{P}$ corresponds to a polynomial of $D+1$ degree with respect to $p$, which is difficult or impossible to be solved in the general case $(D>1)$. A more tractable form of $\hat{P}_{P}, \tilde{P}_{P}$, is analyzed instead. $\tilde{P}_{P}$ is equal to $\frac{1}{N} \sum_{\forall u \in V} \frac{1+p(q-1)}{q} \lambda(1-p \lambda)^{\mid \overline{S \mid}}$, where $\overline{|S|}=\frac{1}{N} \sum_{\forall v \in V}\left|S_{v}\right|$. Finally, $\tilde{P}_{P}$ is given by the following equation.

$$
\tilde{P}_{P}=\frac{1+p(q-1)}{q} \lambda(1-p \lambda)^{\overline{|S|}} \text {. }
$$

$\overline{|S|}$ is the average number of neighbor nodes of each node in the network. Let $\overline{|S|} / D$ be referred to as the topology density for a particular network. Given that $D$ is known (and constant), knowledge of $\overline{|S|}$ is enough to determine the topology density $\overline{|S|} / D$ and vice versa. It is clear that $\overline{|S|}$ (or the topology density $\overline{|S|} / D$ ) influences exponentially the system throughput as it can be concluded from Equation (10).

$\hat{P}_{P}$ may or may not be a good approximation of $P_{P}$, while $\tilde{P}_{P}$ may or may not be a good approximation of $\hat{P}_{P}$. As it can be seen in Section 6, even for those cases when $\hat{P}_{P}$ is not a good approximation of $P_{P}$, and $\tilde{P}_{P}$ is not a good approximation of $\hat{P}_{P}$, the results of the following analysis may be applied.

The following two theorems show that there exists a range of values of $p$ of the form $\left[0, p_{\max }\right]$ (where $p_{\max }, 0<p_{\max } \leq$ 1 , corresponds to that value of $p$ for which $\tilde{P}_{P}=P_{D}$ ) such that $\tilde{P}_{P} \geq P_{D}$, irrespectively of the value of $\lambda$. 
Theorem 1. There exists an efficient range of values for $p$ such that $\tilde{P}_{P} \geq P_{D}$, irrespectively of the value of $\lambda$.

Proof. Under the Deterministic Policy, $q$ is the maximum number of time slots during which a transmission is successful and therefore, $q \lambda$ is the maximum (average) number of successful transmissions in one frame. For any transmission $u \rightarrow v$, according to Equation (5), $q-\left|C_{u \rightarrow v}\right|+\sum_{i \in C_{u \rightarrow v}}(1-\lambda)^{\left|O_{i, u \rightarrow v}\right|} \lambda \leq q \lambda$. Consequently, according to Equation (7), $P_{D} \leq \frac{\lambda}{q}$. It thus suffices to identify the range of values of $p$ for which $\tilde{P}_{P} \geq \frac{\lambda}{q}$.

In Appendix 10 it is shown that $\frac{d \tilde{P}_{P}}{d p}=\frac{p \lambda(\overline{|S|}+1)(1-q)+q-1-\lambda \overline{|S|}}{q} \lambda(1-p \lambda)^{\overline{|S|}-1}$ and therefore, $\frac{d \tilde{P}_{P}}{d p}=0$ for $p=$ $\frac{q-1-\lambda \overline{|S|}}{\lambda(\overline{|S|}+1)(q-1)}\left(\equiv p_{0}\right)$. Given that $\tilde{P}_{P}=P_{D}$ for $p=0$, the required range of values for $p$ does not exist if $\frac{d \tilde{P}_{P}}{d p}<0$ for all $p \in(0,1] \cdot \frac{d \tilde{P}_{P}}{d p}<0$ is satisfied if $p_{0}<0$ and as it is shown in Appendix 10, $p_{0}<0$, if $\lambda>\frac{q-1}{\overline{|S|}}$. Given that $\overline{|S|} \leq D$ (equality holds if all nodes have $D$ neighbor nodes) and $q \geq k D+1 \geq D+1$ (equality holds for $k=1$ ), it is concluded that $\overline{|S|} \leq q-1$ or $\frac{q-1}{\overline{|S|}} \geq 1$. Given that $\lambda \leq 1, \lambda>\frac{q-1}{\overline{|S|}}$ is not satisfied and consequently, it is not possible that $\frac{d \tilde{P}_{P}}{d p}<0$ for all $p \in(0,1]$. Consequently, there exists a range of values for $p$ such that $\tilde{P}_{P} \geq P_{D}$, irrespectively of the value of $\lambda$.

Theorem 2. The efficient range of values for $p$ of Theorem 1 is of the from $\left[0, p_{\max }\right]$, for some $0<p_{\max } \leq 1$.

Proof. If $\lambda \geq \frac{q-1}{q|S|+q-1}$ is satisfied then, according to the Appendix 10, $p_{0} \leq 1$ is satisfied, where $p_{0}=\frac{q-1-\lambda \overline{|S|}}{\lambda(\overline{|S|}+1)(q-1)}$. From Appendix 10 it can be seen that for $p$ close to $0, \frac{d \tilde{P}_{P}}{d p}>0$. Given that $\frac{d \tilde{P}_{P}}{d p}=0$ for $p=p_{0}, \frac{d \tilde{P}_{P}}{d p}>0$ for $p<p_{0}$, and $\frac{d \tilde{P}_{P}}{d p}<0$ for $1 \geq p>p_{0}\left(\frac{d \tilde{P}_{P}}{d p}=0\right.$ for only one value of $p \in(0,1)$ as it may be seen from Appendix 10). As a result, when $\lambda \geq \frac{q-1}{q|S|+q-1}, \tilde{P}_{P}=\frac{\lambda}{q}$ for $p=0$, and as $p$ increases, $\tilde{P}_{P}$ increases, until $p=p_{0}$. As $p$ increases and $p>p_{0}, \tilde{P}_{P}$ decreases until $p=1$, where $\tilde{P}_{P}=\lambda(1-\lambda)^{\overline{|S|}}$. If $\lambda(1-\lambda)^{\overline{|S|}}>\frac{\lambda}{q}$, then $p_{\text {max }}=1$. If $\lambda(1-\lambda)^{\overline{|S|}} \leq \frac{\lambda}{q}$, there exists a value of $p=p_{\max } \leq 1$, such that $\tilde{P}_{P}=\frac{\lambda}{q}$.

If $\lambda<\frac{q-1}{q|S|+q-1}$, then $\frac{d \tilde{P}_{P}}{d p}>0$, for any value of $p$. Consequently, $\tilde{P}_{P}$ constantly increases and therefore, $p_{\max }=1$ and the maximum is assumed for $p=1$.

The example of Figure 4 depicts three possible shapes of the curve corresponding to $\tilde{P}_{P}$. For the case corresponding to $\lambda=1, p_{0}$ and $p_{\max }$ have been drawn as well. For the case corresponding to $\lambda=0.5$, it is clear the $p_{0}$ is close to 0.4 and $p_{\max }=1$. For the case corresponding to $\lambda=0.1$, the maximum is assumed for $p=1$ and $p_{\max }=1$ as well. It is clear that for different values of $\lambda$, different values of $p$ correspond to $\tilde{P}_{P}$ maximization. Theorem 3 establishes the conditions and presents the relations for the system throughput maximization.

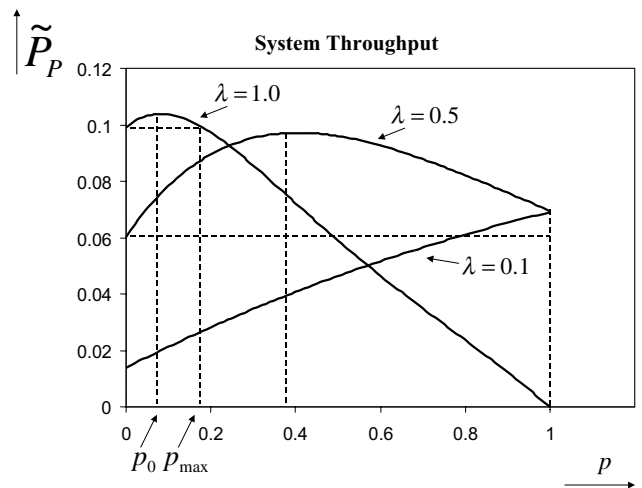

Fig. 4. $\tilde{P}_{P}$ as a function of $p$ for different value of $\lambda$.

Theorem 3. If $\lambda<\frac{q-1}{q \overline{|S|}+q-1}$, $\tilde{P}_{P}$ is maximized for $p=1$. If $\lambda \geq \frac{q-1}{q \mid \overline{|S|}+q-1}$, $\tilde{P}_{P}$ is maximized for $p=\frac{q-1-\lambda \overline{|S|}}{\lambda(\overline{|S|}+1)(q-1)}$.

Proof. For $\lambda<\frac{q-1}{q|S|+q-1}$, as it was shown in the proof of Theorem $2, \frac{d \tilde{P}_{P}}{d p}>0$. Consequently, the maximum value for $\tilde{P}_{P}$ is assumed for $p=1$.

For $\lambda \geq \frac{q-1}{q|S|+q-1}$, as it was shown in the proof of Theorem $2, \frac{d \tilde{P}_{P}}{d p} \geq 0$ for $p \in\left[0, p_{0}\right]$ and $\frac{d \tilde{P}_{P}}{d p} \leq 0$ for $p \in\left[p_{0}, 1\right]$. Consequently, the maximum value for $\tilde{P}_{P}$ is assumed for $p=p_{0}=\frac{q-1-\lambda \overline{S \mid}}{\lambda(\overline{|S|}+1)(q-1)}$. 
Based on the results of Theorem 3 , the value of the access probability $p$ that maximizes $\tilde{P}_{P}$, denoted by $\tilde{p}_{\lambda,|S|}$, is given by the following equation.

$$
\tilde{p}_{\lambda, \overline{|S|}}=\left\{\begin{aligned}
& 1, \text { if } \lambda<\frac{q-1}{q|S|+q-1} \\
& \frac{q-1-\lambda \overline{|S|}}{\lambda(\overline{|S|}+1)(q-1)}, \text { if } \lambda \geq \frac{q-1}{q \overline{|S|}+q-1}
\end{aligned}\right.
$$

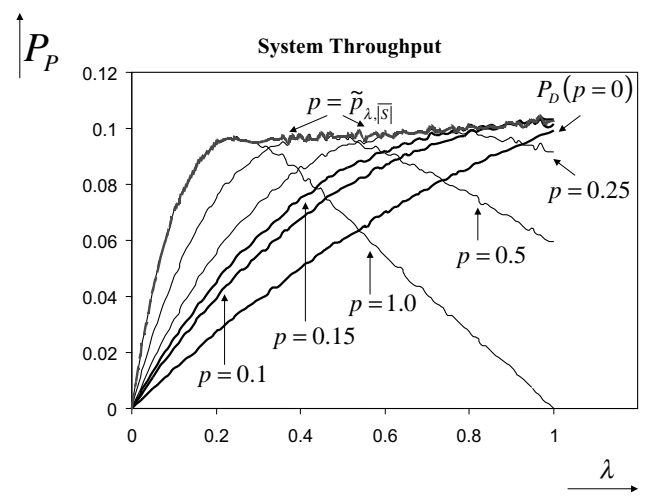

Fig. 5. Simulation results for the system throughput $\left(P_{P}\right)$ under the Probabilistic Policy as a function of $\lambda$, for different values of $p\left(p=0, p=0.1, p=0.15, p=0.25, p=0.5, p=1.0\right.$ and $\left.p=\tilde{p}_{\lambda,|S|}\right) . \overline{|S|}=2.963$ and $\overline{|S|} / D=0.494$.

For $p=\tilde{p}_{\lambda,|S|}, \tilde{P}_{P}$ is maximized but it is also expected that $P_{P}$ will have a better performance than that achieved by a fixed value of $p$ when $\lambda$ is not constant. This is illustrated in Figure 5 concerning simulation results (more information about the simulator can be found in Section 8) for the network depicted in Figure 1. In this figure, $P_{P}$ for $p=\tilde{p}_{\lambda, \mid \overline{S \mid}}$ is close to the maximum obtained by any other curve for constant values of $p$. The cases under which the results of this analysis may be used to maximize $P_{P}$ (as opposed to $\tilde{P}_{P}$ that has been examined in the present section), are investigated in the following section.

\section{On the Accuracy of the Approximation}

How close is $\hat{P}_{P}$ to $P_{P}$ depends on how close is $\frac{1-\lambda}{1-p \lambda}$ to 1 . Obviously, $\frac{1-\lambda}{1-p \lambda}$ is close to 1 for small values of $\lambda$ and large values of $p$, as it can be seen in Appendix 11. This is clearly depicted in Figure 6, concerning the network depicted in Figure 1, where $P_{P}$ and $\hat{P_{P}}$ are depicted (a) as a function of $\lambda$ for different values of $p$ and (b) as a function of $p$ for different values of $\lambda$. The curve corresponding to $\hat{P}_{P}$ is very close to the one corresponding to $P_{P}$ for small values of $\lambda$ and/or large values of $p$.

An interesting observation on Figure 6 is that those values of $p$ and $\lambda$, whose curves corresponding to $P_{P}$ and $\hat{P}_{P}$ are not close, have almost the same shape. This means that those values of $p$ and $\lambda$, for which $\hat{P}_{P}$ assumes the maximum, are close to the value of $p$ and $\lambda$ for which $P_{P}$ assumes the maximum, as it may also be observed from Figure 6 .

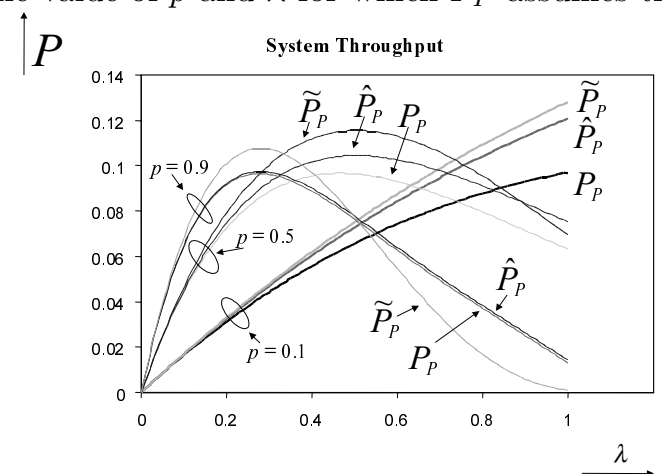

(a)

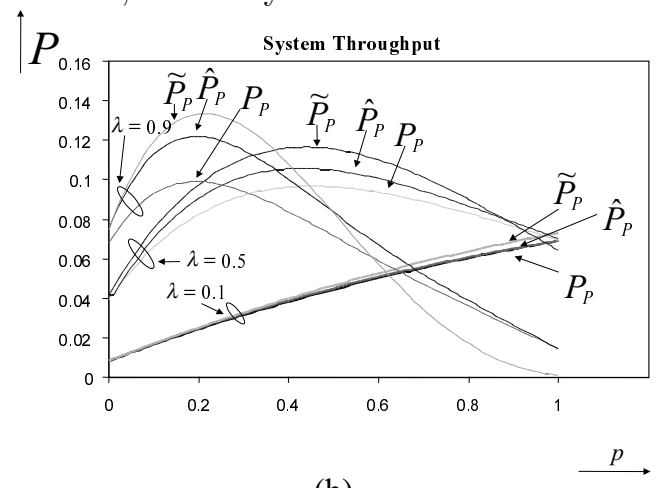

(b)

Fig. 6. Numerical results for the system throughput $(P)$ corresponding to $P_{P}, \hat{P}_{P}$ and $\tilde{P}_{P}$, (a) as a function of $\lambda$, for different values of $p(p=0.1, p=0.5$ and $p=0.9)$ and (b) as a function of $p$, for different values of $\lambda(\lambda=0.1, \lambda=0.5$ and $\lambda=0.9)$. Note that for $p=0, P_{P}=P_{D}$.

How close is $\hat{P}_{P}$ to $\tilde{P}_{P}$ depends on how close is $\left|\tilde{P}_{P}-\hat{P}_{P}\right|$ to 0 . In Appendix 12 it is shown that $\left|\tilde{P}_{P}-\hat{P}_{P}\right|=$ $\frac{1+p(q-1)}{q} \lambda(1-p \lambda)^{\overline{|S|}}\left|\frac{1}{N} \sum_{\forall u \in V}(1-p \lambda)^{\left|S_{v}\right|-\overline{|S|}}-1\right|$. Let $\operatorname{Var}\{|S|\}=\frac{1}{N D} \sum_{v \in V}|\overline{|S|}-| S_{v}||$ be defined as the topology 
density variation. It is evident that as $\operatorname{Var}\{|S|\}$ increases, $\left|\tilde{P}_{P}-\hat{P}_{P}\right|$ increases exponentially. Consequently, $\tilde{P}_{P}$ is a good approximation of $\hat{P}_{P}$ for rather small values of $\operatorname{Var}\{|S|\}$. In the exceptional case of a network for which all nodes have the same number of neighbor nodes (except perhaps the particular node that has $D$ neighbor nodes), $\tilde{P}_{P} \approx \hat{P}_{P}$ $(\operatorname{Var}\{|S|\} \rightarrow 0)$. For the case that all nodes have equal number of neighbor nodes and equal to $D$, then $\tilde{P}_{P}=\hat{P}_{P}$ $(\operatorname{Var}\{|S|\}=0)$. An example of the latter case is a network with uniformly distributed nodes on a sphere's surface. For the example network depicted in Figure 1 it can be calculated that $\operatorname{Var}\{|S|\}=0.364$.

In Figure 6, where $\tilde{P}_{P}$ is also depicted, it can be seen that the shape of the corresponding curve is similar to that of the curve corresponding to $\hat{P}_{P}$. It can also be seen that for small values of $p$ and $\lambda$ both curves are close. This holds since $\left|\frac{1}{N} \sum_{\forall u \in V}(1-p \lambda)^{\left|S_{v}\right|-\overline{|S|}}-1\right|$ is close to zero (and consequently $\left|\tilde{P}_{P}-\hat{P}_{P}\right|$ is close to zero) for small values of $p$ and $\lambda$. It is evident that even though $\hat{P}_{P}$ may not be close to $\tilde{P}_{P}$, for a given $\lambda$ the values of $p$ for which $\tilde{P}_{P}$ is maximized are close to the values of $p$ for which $\hat{P}_{P}$ is maximized.

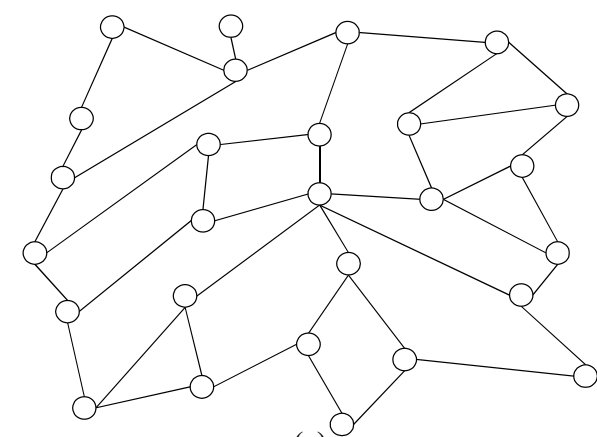

(a)

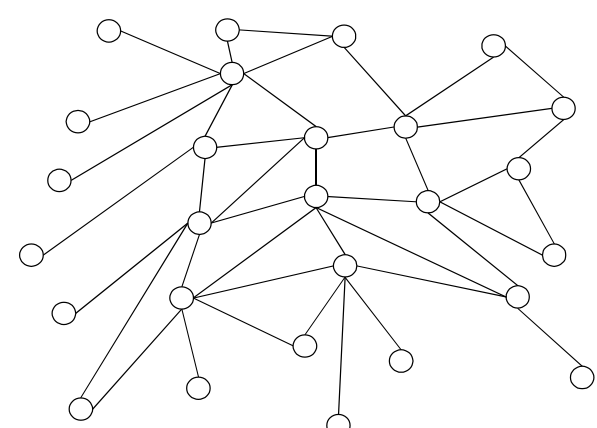

(b)

Fig. 7. Networks $A$ (a) and $B($ b) with the same topology density value $(\overline{|S|} / D=0.494)$ with Network $C$ (Figure 1$)$ but different topology density variation $(\operatorname{Var}\{|S|\}=0.037,0.198$ and 0.136 , respectively).

In order to provide an example let the networks depicted in figures $7(\mathrm{a}), 7(\mathrm{~b})$ and 1 be referred to as network $A, B$ and $C$, respectively. All three networks have the same number of nodes $(N=27)$, the same number of maximum neighbor nodes $(D=6)$ and the same topology density $(\overline{|S|} / D=0.494)$.

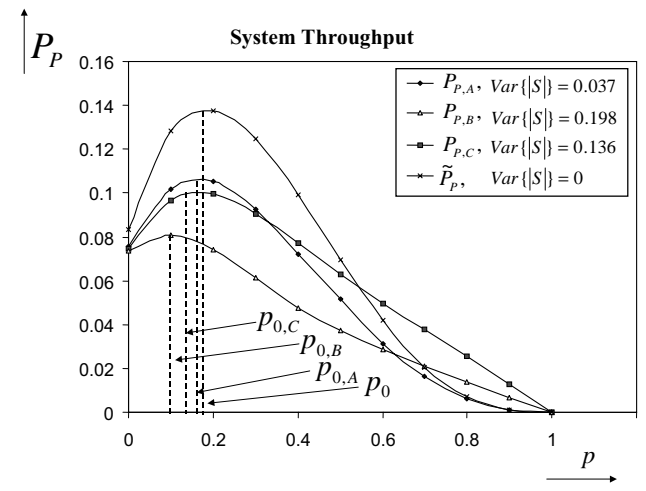

Fig. 8. System throughput for network $A, B$ and $C$.

Figure 8 depicts $P_{P, A}, P_{P, B}$ and $P_{P, C}$ for the three aforementioned networks as well as $\tilde{P}_{P}$ as a function of $p$ and for $\lambda=1$. These are simulation results and were obtained as it is described in Section 8. Let $p_{0, A}, p_{0, B}$ and $p_{0, C}$ those values of $p$ for which $P_{P, A}, P_{P, B}$ and $P_{P, C}$ are maximized, respectively. It is observed from Figure 8 that as $\operatorname{Var}\{|S|\}$ increases the difference between $P_{P}$ (for any of the networks) and $\tilde{P}_{P}$ increases, while the difference between the value of $p$ that maximizes $P_{P}$ and the value of $p$ that maximizes $\tilde{P}_{P}\left(p_{0}\right)$, increases but not dramatically.

From the previous discussion it is concluded that the smaller the value of $\lambda$ and $\operatorname{Var}\{|S|\}$ and the higher the value of $p$, the better the approximation of $P_{P}$ by $\tilde{P}_{P}$. However, even for those cases that $P_{P}$ is not too close to $\tilde{P}_{P}$, the results of the analysis for $\tilde{P}_{P}$ may be used in order to achieve system throughput, $P_{P}$, close to the maximum.

\section{Unawareness of $\lambda$ and $\overline{|S|}$}

Knowledge of both $\lambda$ and $\overline{|S|}$ is required in order to utilize the results of the analysis presented in Section 5 and in particular, by Equation (11). If the topology density $\overline{|S|} / D$ is known, $\overline{|S|}$ can be calculated ( $D$ is known). In the general case, it is possible that $\lambda$ and/or $\overline{|S|}$ are not known and therefore, it would be useful to derive analytical expressions for 
those values of $p$ that the system throughput under the Probabilistic Policy even though it is not maximized, at least is higher than that under the Deterministic Policy.

For the case for which $\lambda$ is known but $\overline{|S|}$ is not, the maximum topology density value (corresponding to $\overline{|S|}=D$ ) is considered and the corresponding value for $p$, denoted by $\tilde{p}_{\lambda}$, is given by Equation (12). For the case for which $\overline{|S|}$ is known but $\lambda$ is not, a heavy traffic scenario (corresponding to $\lambda=1$ ) is considered and the corresponding value for $p$, denoted by $\tilde{p}_{\overline{|S|} \mid}$, is given by Equation (13). Finally, for the case for which both $\lambda$ and $\overline{|S|}$ are not known, the corresponding value for $p(\lambda=1$ and $\overline{|S|}=D)$, denoted by $\tilde{p}$, is given by Equation (14).

$$
\begin{aligned}
& \tilde{p}_{\lambda}=\left\{\begin{array}{r}
1, \text { if } \lambda<\frac{q-1}{q D+q-1} \\
\frac{q-1-\lambda D}{\lambda(D+1)(q-1)}, \text { if } \lambda \geq \frac{q-1}{q D+q-1}
\end{array}\right. \\
& \tilde{p}_{\overline{|S|}}=\frac{q-1-\overline{|S|}}{(\overline{|S|}+1)(q-1)} \\
& \tilde{p}=\frac{q-1-D}{(D+1)(q-1)} .
\end{aligned}
$$

The following theorem shows that for any value of $p$ derived from any of the equations (12), (13) and (14), the system throughput under the Probabilistic Policy is higher than that under the Deterministic Policy.

Theorem 4. For any $p$ equal to $\tilde{p}_{\lambda}$, $\tilde{p}_{\overline{|S|}}$ or $\tilde{p}, \tilde{P}_{P} \geq P_{D}$ is satisfied.

Proof. $\tilde{p}_{\lambda, \overline{|S|}}$ belongs to the efficient range of values for $p$ for which $\tilde{P}_{P} \geq P_{D}$ is satisfied. According to Theorem 2 , this range of values is of the form $\left[0, p_{\max }\right]$, for some $0<p_{\max } \leq 1$. It is is enough to show that $\tilde{p}_{\lambda}$, $\tilde{p}_{\overline{|S|}}$ and $\tilde{p}$ are less or equal to $\tilde{p}_{\lambda, \overline{|S|}}$.

In order to show that $\tilde{p}_{\lambda} \leq \tilde{p}_{\lambda, \overline{|S|}}$, both ranges of values for $\lambda$ of equations (11) and (12) should be compared. Given that $\overline{|S|} \leq D, \frac{q-1}{q D+q-1} \leq \frac{q-1}{q \mid \overline{S \mid}+q-1}$. Consequently, for $\lambda<\frac{q-1}{q D+q-1}$, according to equations (11) and (12), the equality $\tilde{p}_{\lambda}=\tilde{p}_{\lambda,|S|}=1$ holds. For $\frac{q-1}{q D+q-1} \leq \lambda<\frac{q-1}{q|S|+q-1}, \tilde{p}_{\lambda, \overline{|S|}}=1$ and $\tilde{p}_{\lambda}=\frac{q-1-\lambda D}{\lambda(D+1)(q-1)}$. As it can be seen in Appendix 13, $\frac{q-1-\lambda D}{\lambda(D+1)(q-1)} \leq 1$ is satisfied and consequently, $\tilde{p}_{\lambda} \leq \tilde{p}_{\lambda,|S|}$ is also satisfied. For $\lambda \geq \frac{q-1}{q|S|+q-1}, \tilde{p}_{\lambda, \overline{|S|}}=\frac{q-1-\lambda \overline{|S|}}{\lambda(\overline{|S|}+1)(q-1)}$ and $\tilde{p}_{\lambda}=\frac{q-1-\lambda D}{\lambda(D+1)(q-1)}$. According to Appendix $13 \frac{q-1-\lambda D}{\lambda(D+1)(q-1)} \leq \frac{q-1-\lambda \mid \overline{S \mid}}{\lambda(\overline{|S|}+1)(q-1)}$, is satisfied and consequently, $\tilde{p}_{\lambda} \leq \tilde{p}_{\lambda, \overline{|S|}}$ is always satisfied.

In order to show that $\tilde{p}_{\overline{|S|}} \leq \tilde{p}_{\lambda, \overline{|S|}}$ is satisfied, it is enough to show that $\frac{q-1-\overline{|S|}}{(\overline{|S|}+1)(q-1)} \leq \frac{q-1-\lambda \overline{|S|}}{\lambda(\overline{|S|}+1)(q-1)}$ is satisfied. Consequently, it is enough to show that $q-1-\overline{|S|} \leq \frac{q-1-\lambda \overline{|S|}}{\lambda}$ or $\lambda(q-1)-\lambda \overline{|S|} \leq q-1-\lambda \overline{|S|}$ or $\lambda(q-1) \leq q-1$. The latter is always satisfied and consequently, $\tilde{p}_{|S|} \leq \tilde{p}_{\lambda, \overline{S \mid}}$.

In order to show that $\tilde{p} \leq \tilde{p}_{\lambda, \overline{|S|}}$ is satisfied, it is enough to show that $\tilde{p} \leq \tilde{p}_{\overline{|S|}}$ is satisfied or $\frac{q-1-D}{(D+1)(q-1)} \leq \frac{q-1-\overline{|S|}}{(\overline{|S|}+1)(q-1)}$ or $\frac{q-1-D}{D+1} \leq \frac{q-1-\overline{|S|}}{\overline{|S|}+1}$ or $(\overline{|S|}+1)(q-1-D) \leq(D+1)(q-1-\overline{|S|})$ or $q \overline{|S|}+q-\overline{|S|}-1-D \overline{|S|}-D \leq q D+q-D-1-\overline{|S|} D-\overline{|S|}$ or $q \overline{|S|} \leq q D$. The latter is always satisfied.

For the heavy traffic scenario $(\lambda=1)$, an approximate analysis was also presented in [17] and boundaries $\left(\tilde{p}_{0_{m i n}}<\right.$ $\tilde{p}_{0_{\text {max }}}$ ) for the value of $p$ that maximizes the system throughput were derived. In particular, it was derived that $\tilde{p}_{0_{\text {min }}}=$ $\frac{q^{2}-(2 \mid \overline{S \mid}+1)\left(q-\frac{2|S|+1}{4}\right)}{\left(q^{2}-(\overline{|S|}+1)\left(q-\frac{2 \overline{S \mid}+1}{4}\right)\right)(\overline{|S|}+1)}$ and $\tilde{p}_{0_{\max }}=\frac{1}{\mid \overline{S \mid}+1}$. As it can be seen in Appendix $14, \tilde{p}_{\overline{|S|}}<\tilde{p}_{0_{\max }}$ is always satisfied and

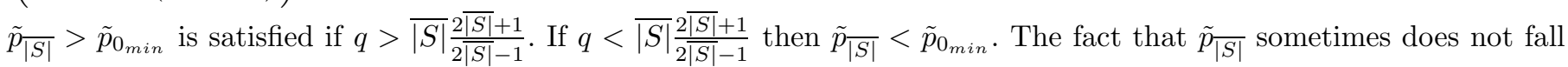
within the boundaries provided by the analysis in [17], is attributed to the accuracy of the different approximations considered in each analysis. Boundaries $\tilde{p}_{0_{\min }}$ and $\tilde{p}_{0_{\max }}$ have been derived based on approximations that their accuracy depends only on $\operatorname{Var}\{|S|\}$, while $\tilde{p}_{\overline{|S|}}$ 's accuracy depends additionally on how close is $\frac{1-\lambda}{1-p \lambda}$ to 1 . Given that for this case $\lambda=1, \frac{1-\lambda}{1-p \lambda}=0$; that is the worst case. Consequently, for those cases for which $\lambda=1$, $\tilde{p}_{0_{\text {min }}}$ can be a better approximation than $\tilde{p}_{|S|}$.

\section{Simulation Results}

Simulation results have been provided already in Section 4 for the example network of 27 nodes depicted in Figure 1. In this section, networks of 100 nodes are considered for various values of $D$ and the topology density $\overline{|S|} / D$. The aim 
is to demonstrate the applicability of the analytical results for a variety of topologies with different characteristics. In particular, four different topology categories are considered. The number of nodes in each topology category is set to $N=100$, while $D$ is set to $5,10,15$ and 20 . These four topology categories are denoted as D5N100, D10N100, D15N100 and D20N100, respectively. Three different topologies that correspond to different topology density values $\overline{|S|} / D$ are considered for each topology category. Figures 9,10 and 11 depict simulation results for the system throughput $P_{P}$, for different topology density values. In particular, in Figure $9, \overline{|S|} / D$ is small (around 0.2 ), in Figure $10, \overline{|S|} / D$ is around 0.6, while in Figure 11, $\mid \overline{|S|} / D$ is high (around 0.85). For all cases the number of neighbor nodes for each node is not the same; this leads to nonzero values for the topology density variation $\operatorname{Var}\{|S|\}$.

The algorithm presented in [14] is used to derive the sets of scheduling slots and the system throughput is calculated averaging the simulation results over 100 frames. Time slot sets $\Omega_{\chi}$ are assigned randomly to each node $\chi$, for each particular topology. The particular assignment is kept the same for each topology category throughout the simulations.

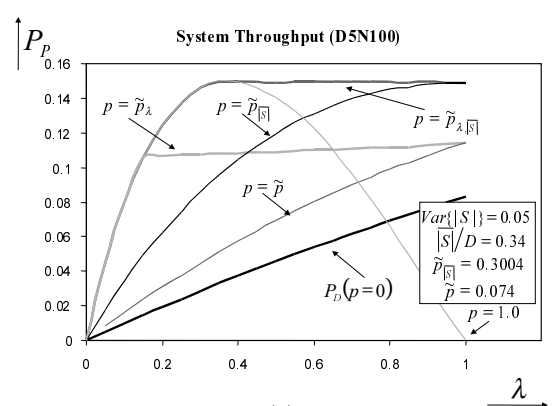

(a)

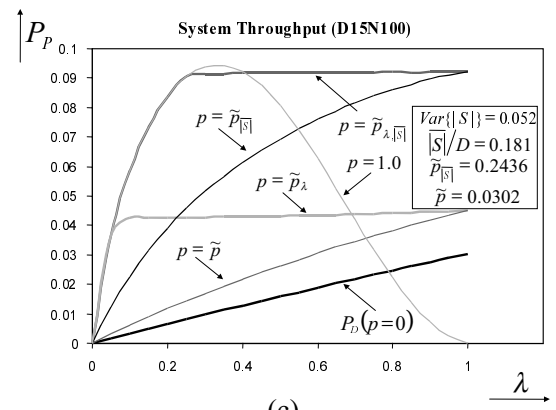

(c)

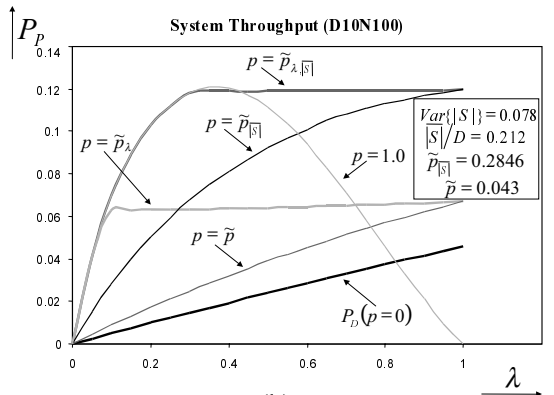

(b)

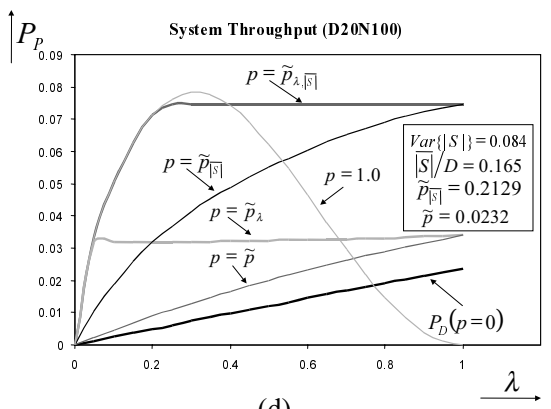

(d)

Fig. 9. System throughput simulation results as a function of $\lambda$ for different values of $p\left(p=0, p=\tilde{p}_{\lambda, \overline{|S|} \mid}, p=\tilde{p}_{\lambda}, p=\tilde{p}_{|S|}, p=\tilde{p}\right.$ and $p=1.0)$ small topology density values $\overline{|S|} / D$.

Figures 9, 10 and 11 depict simulation results for the system throughput $P_{P}$ as a function of $\lambda$, for different values of $p\left(p=0, p=\tilde{p}_{\lambda,|S|}, p=\tilde{p}_{\lambda}, p=\tilde{p}_{\overline{|S|} \mid}, p=\tilde{p}\right.$ and $\left.p=1.0\right)$. For $p=0$ the system throughput under the Probabilistic Policy is identical to that under the Deterministic Policy $\left(P_{P}=P_{D}\right)$. This case is depicted throughout the simulation results for comparison reasons.

For $p=1.0$, it can be seen that as $\lambda$ increases, $P_{P}$ increases rather fast until a certain maximum value and then decreases until $\lambda=1.0$, where $P_{P}=0$. As it can be observed from the three figures $(9,10$ and 11), as the topology density increases, the value of $\lambda$ for which $P_{P}$ assumes the maximum, decreases.

For $p=\tilde{p}_{\lambda, \overline{S \mid}}$ it is evident that the system throughput under the Probabilistic Policy is not only higher than that under the Deterministic Policy but it is also close to the maximum for all different topologies.

For $p=\tilde{p}_{\lambda}$ and for small values of $\lambda$, as it can be seen in Figure 9, the system throughput is identical to that obtained for $p=\tilde{p}_{\lambda, \overline{S \mid}}$ and $p=1.0$. This can be concluded from Equation (11) and Equation (12) where for $\lambda<\frac{q-1}{q D+q-1}$, $\tilde{p}_{\lambda, \overline{|S|}}=\tilde{p}_{\lambda}=1.0$. For $\lambda>\frac{q-1}{q D+q-1}$ the system throughput, even though not close to that obtained for $p=\tilde{p}_{\lambda,|S|}$, is higher than that obtained under the Probabilistic Policy. As the topology density increases (Figure 10 and Figure 11) it is clear that the system throughput obtained for $p=\tilde{p}_{\lambda}$ is close to the maximum.

For $p=\tilde{p}_{\overline{|S|}}$ and for small values of $\lambda$, the system throughput is not close to that obtained for $p=\tilde{p}_{\lambda,|S|}$ but is higher than that obtained under the Deterministic Policy, irrespectively of the topology density value. As $\lambda$ increases the system throughput increases and for large values of $\lambda$ it is close to the maximum.

For $p=\tilde{p}$, the system throughput curve is similar to that for $p=\tilde{p}_{\overline{|S|} \mid}$, except that the obtained system throughput is smaller. As the topology density increases, the system throughput for both cases $\left(p=\tilde{p}\right.$ and $\left.p=\tilde{p}_{|S|}\right)$ converges. Again, the obtained system throughout is higher than that obtained under the Deterministic Policy. This is an important observation since $\tilde{p}$ is calculated without knowledge of either $\lambda$ or $\overline{|S|}$. 


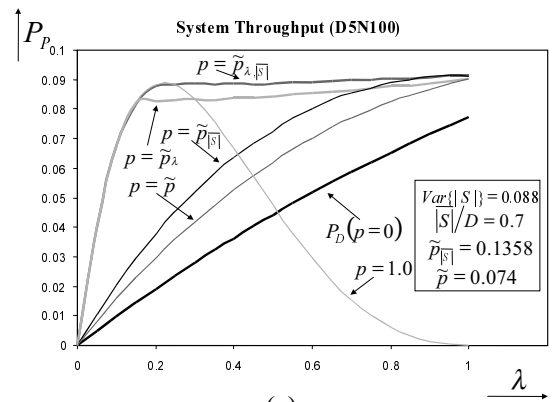

(a)

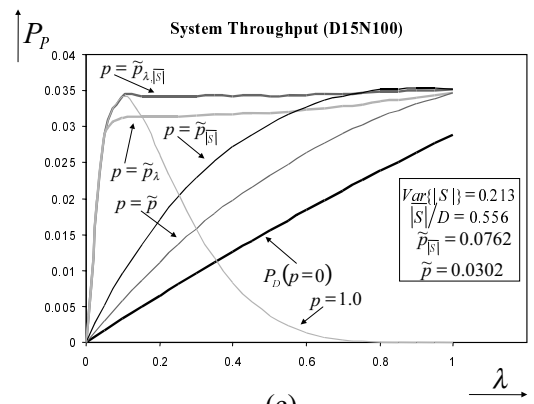

(c)

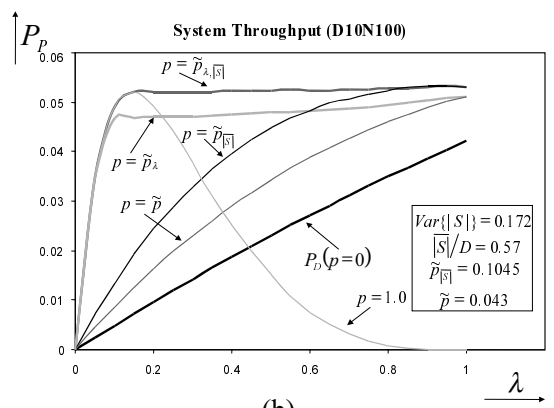

(b)

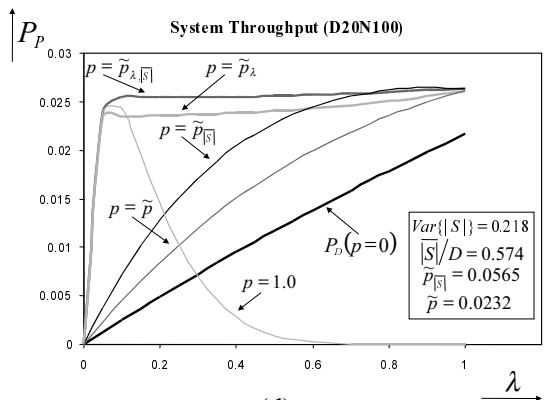

(d)

Fig. 10. System throughput simulation results as a function of $\lambda$ for different values of $p\left(p=0, p=\tilde{p}_{\lambda, \overline{S \mid}}, p=\tilde{p}_{\lambda}, p=\tilde{p}_{|S|}\right.$, $p=\tilde{p}$ and $p=1.0)$ and medium topology density values $\overline{|S|} / D$.

In order to obtain a clearer view, the system throughput for the D10N100 topology category and topology density $\overline{|S|} / D=0.57$ and $\operatorname{Var}\{|S|\}=0.172$, is depicted in Figure 12 as a function of $p$, for $\lambda=0.1, \lambda=0.3, \lambda=0.6$ and $\lambda=0.9$. It is evident that for $p=\tilde{p}_{\lambda, \overline{|S|}}$, the system throughput assumes the maximum value for any value of $\lambda$. For any other case $\left(p=\tilde{p}_{\lambda}, p=\tilde{p}_{|S|}\right.$ or $\left.p=\tilde{p}\right)$ the system throughput is less than that obtained for $p=\tilde{p}_{\lambda,|S|}$ but definitely higher than that obtained under the Deterministic Policy. The latter was expected as a direct result from Theorem 4 . In all cases it is evident that the system throughput achieved under the Probabilistic Policy is higher than achieved under the Deterministic Policy.

Another factor that influences the system throughput is the topology density $\overline{|S|} / D$, as it can be seen from the exponential factor of Equation (10). In Figure 13, it can be seen that the system throughout under the Deterministic Policy decreases slowly as $\overline{|S|} / D$ increases, while the system throughout under the Probabilistic Policy decreases rapidly as $\overline{|S|} / D$ increases. The only exception is for $p=\tilde{p}$, where the decreament is not so rapid. It can also be observed that as $\overline{|S|} / D$ increases, the curves corresponding to $p=\tilde{p}_{\lambda,|S|}$ and $p=\tilde{p}_{\lambda}$ converge as well as those corresponding to $p=\tilde{p}_{\overline{|S|}}$ and $p=\tilde{p}$. For large values of $\lambda$ and $\overline{|S|} / D$ the four aforementioned curves converge to the same value. The reason is, according to equations (11), (12), (13) and (14), that the values of $\tilde{p}_{\lambda, \overline{|S|}}, \tilde{p}_{\lambda}, \tilde{p}_{\overline{|S|}}$ and $\tilde{p}$ converge to the same value, for $\lambda$ close to 1 and $\overline{|S|}$ close to $D$.

\section{Conclusions}

The system throughput under both the Deterministic Policy and the Probabilistic Policy has been investigated previously for heavy traffic conditions, [13], [14], [15], [16], [17]. In this work, expressions have been derived and analyzed for the general non-heavy traffic case. The results of this analysis established conditions and provided expressions in order for the system throughput under the Probabilistic Policy to be not only higher than that under the Deterministic Policy, but also be maximized, for various traffic loads.

Expressions for the system throughput were derived for both policies as a function of the traffic load and the accuracy of certain analysis approximations was investigated. These derived expressions determine the values of the access probability $p$ for which the system throughput under the Probabilistic Policy is higher than that under the Deterministic Policy. For the particular case for which both the traffic load $(\lambda)$ and the topology density $(\overline{|S|} / D)$ are known, the system throughput is maximized for the derived value of access probability, $p=\tilde{p}_{\lambda, \overline{|S|}}$. For the cases for which $\lambda$ and/or $\overline{|S|} / D$ are not known, analytical expressions for the appropriate values of $p$ were also derived leading to a system throughput which, even though not maximized, is higher than that achieved under the Deterministic Policy $\left(p=\tilde{p}_{\lambda}, p=\tilde{p}_{|S|}\right.$ and $p=\tilde{p})$. 


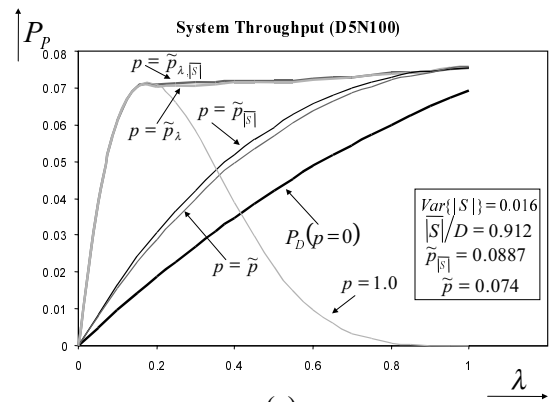

(a)

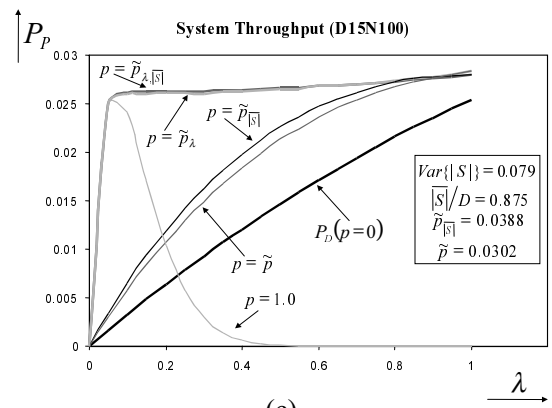

(c)

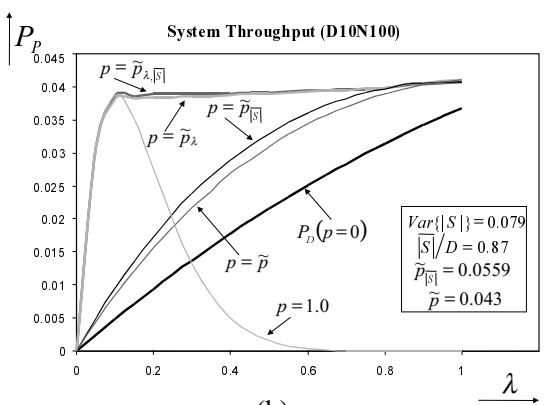

(b)

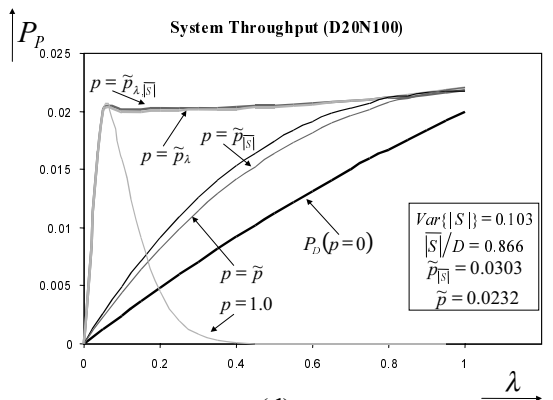

(d)

Fig. 11. System throughput simulation results as a function of $\lambda$ for different values of $p\left(p=0, p=\tilde{p}_{\lambda, \overline{S \mid}}, p=\tilde{p}_{\lambda}, p=\tilde{p}_{|S|}\right.$, $p=\tilde{p}$ and $p=1.0)$ and high topology density values $\overline{|S|} / D$.

Simulations have been conducted for a variety of different topologies with different characteristics and different traffic loads that support the claims and the expectations of the analysis. The simulation results have shown that the achieved system throughput is maximized, provided that $\lambda$ and $\overline{|S|} / D$ are known. If the latter is not the case, the system throughput can still be higher than that under the Deterministic Policy provided that the results of the aforementioned analysis are applied. The simulation results also show how the system throughput is affected by the topology density $\overline{|S|} / D$ and demonstrate the fact that the use of the analytical results assures a system throughput under the Probabilistic Policy higher than that under the Deterministic Policy even for large values of $\overline{|S|} / D$.

In conclusion, a simple and easily implemented transmission policy like the Probabilistic Policy, allows for nodes to transmit without any need for coordination among them and without considering the topological changes; this is essential for ad-hoc networks. The results of the analysis provided in this paper can be used to specify an access probability that achieves a system throughput close to the maximum for different traffic loads.

\section{Acknowledgement}

This work has been supported in part by the IST program under contract IST-2001-32686 (BroadWay) which is partly funded by the European Commission.

\section{References}

1. IEEE 802.11, "Wireless LAN Medium Access Control (MAC) and Physical Layer (PHY) specifications," Nov. 1997. Draft Supplement to Standard IEEE 802.11, IEEE, New York, January 1999.

2. P. Karn, "MACA- A new channel access method for packet radio," in ARRL/CRRL Amateur Radio 9th Computer Networking Conference, pp. 134-140, 1990.

3. V. Bharghavan, A. Demers, S. Shenker, and L. Zhang, "MACAW: A Media Access Protocol for Wireless LAN's," Proceedings of ACM SIGCOMM'94, pp. 212-225, 1994.

4. C.L. Fullmer, J.J. Garcia-Luna-Aceves, "Floor Acquisition Multiple Access (FAMA) for Packet-Radio Networks," Proceedings of ACM SIGCOMM'95, pp. 262-273, 1995.

5. J. Deng and Z. J. Haas, "Busy Tone Multiple Access (DBTMA): A New Medium Access Control for Packet Radio Networks," in IEEE ICUPC'98, Florence, Italy, October 5-9, 1998.

6. R. Nelson, L. Kleinrock, "Spatial TDMA, A collision-free Multihop Channel Access Protocol", IEEE Transactions on Communications, Vol. COM-33, No. 9, September 1985.

7. A. Ephremides and T. V. Truong, "Scheduling Broadcasts in Multihop Radio Networks," IEEE Transactions on Communications, 38(4):456-60, April 1990. 


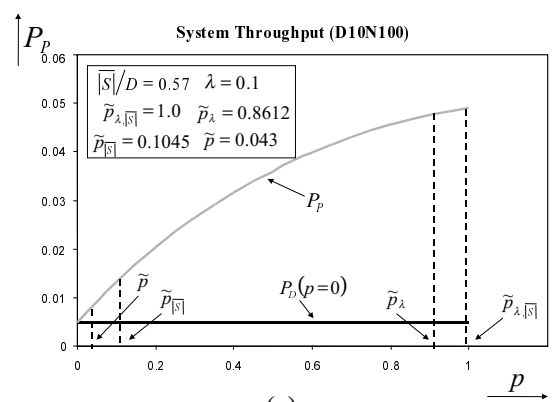

(a)

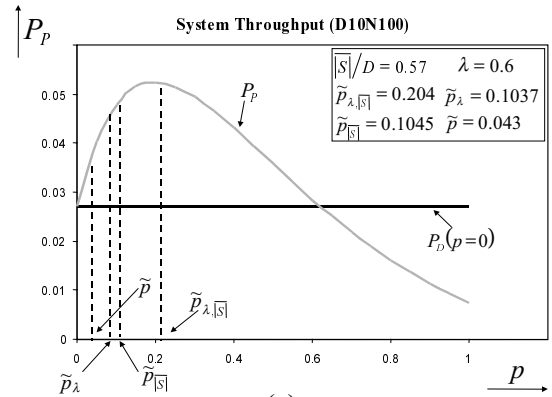

(c)

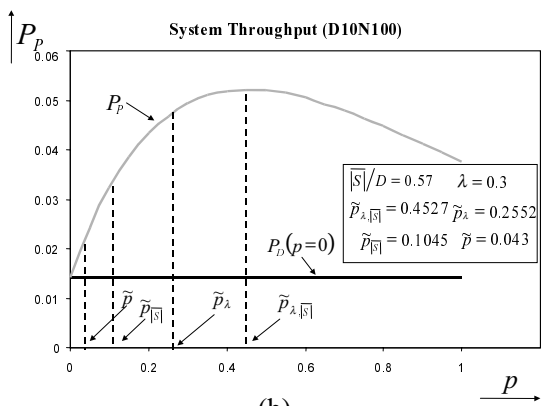

(b)

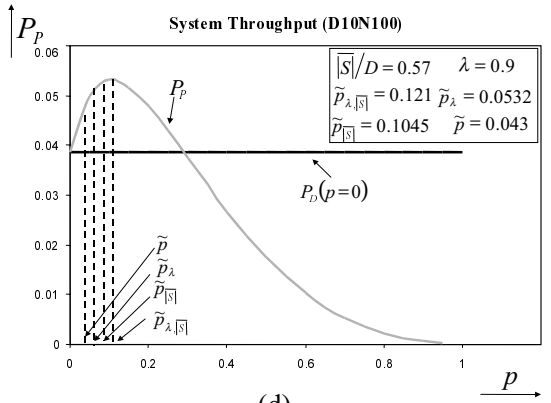

(d)

Fig. 12. System throughput simulation results for the D10N100 topology $(\overline{|S|} / D=0.57$ and and $\operatorname{Var}\{|S|\}=0.172)$ as a function of $p$ for different values of $\lambda(\lambda=0.1, \lambda=0.3, \lambda=0.6$ and $\lambda=0.9)$.

8. G. Wang and N. Ansari, "Optimal Broadcast Scheduling in Packet Radio Networks Using Mean Field Annealing," IEEE Journal on Selected Areas in Communications, VOL. 15, NO. 2, pp 250-260, February 1997.

9. T. Shepard, "A Channel Access Scheme for Large Dense Packet Radio Networks," In Proc. of ACM SIGCOMM (Aug. 1998).

10. L. Bao and J. J. Garcia-Luna-Aceves, "A new approach to channel access scheduling for ad hoc networks," ACM Mobicom 2001, July 2001.

11. R. Rozovsky and P. R. Kumar, "SEEDEX: A MAC protocol for ad hoc networks," ACM Mobihoc'01, October 2001.

12. F. Borgonovo, A. Capone, M. Cesana, L. Fratta, "ADHOC MAC: a new MAC architecture for ad hoc networks providing efficient and reliable point-to-point and broadcast services," to appear in WINET Special Issue on Ad Hoc Networking.

13. I. Chlamtac and A. Farago, "Making Transmission Schedules Immune to Topology Changes in Multi-Hop Packet Radio Networks," IEEE/ACM Trans. on Networking, 2:23-29, 1994.

14. J.-H. Ju and V. O. K. Li, "An Optimal Topology-Transparent Scheduling Method in Multihop Packet Radio Networks," IEEE/ACM Trans. on Networking, 6:298-306, 1998.

15. K. Oikonomou and I. Stavrakakis, "A Probabilistic Topology Unaware TDMA Medium Access Control Policy for Ad-Hoc Environments," Personal Wireless Communications (PWC 2003), 23-25 September, 2003, Venice, Italy.

16. K. Oikonomou and I. Stavrakakis, "Throughput Analysis of a Probabilistic Topology-Unaware TDMA MAC Policy for Ad-Hoc Networks," Quality of Future Internet Services (QoFIS), 1-3 October, 2003, Stockholm, Sweden.

17. K. Oikonomou and I. Stavrakakis, "Analysis of a Probabilistic Topology-Unaware TDMA MAC Policy for Ad-Hoc Networks," IEEE Journal on Selected Areas in Communications (JSAC), Special Issue on Quality-of-Service Delivery in Variable Topology Networks. Accepted for publication. To appear 3rd-4th Quarter 2004.

18. J. A. Stankovic, T. Abdelzaher, C. Lu, L. Sha, J. Hou, "Real-Time Communication and Coordination in Embedded Sensor Networks," Proceedings of the IEEE, 91(7): 1002-1022, July 2003.

19. R. Krishnan and J.P.G. Sterbenz, "An Evaluation of the TSMA Protocol as a Control Channel Mechanism in MMWN," Technical report, BBN Technical Memorandum No. 1279, 2000.

20. Dimitri Bertsekas and Robert Gallager, "Data networks," 2nd edition, Prentice-Hall, Inc., 1992.

\section{First Derivative $\frac{d \tilde{P}_{P}}{d p}$}

$$
\frac{d \tilde{P}_{P}}{d p}=\frac{p \lambda(\overline{|S|}+1)(1-q)+q-1-\lambda \overline{|S|}}{q} \lambda(1-p \lambda)^{\overline{|S|}-1} .
$$

Obviously, $\frac{d \tilde{P}_{P}}{d p}=0$ for $p=\frac{q-1-\lambda \overline{|S|}}{\lambda(\overline{|S|}+1)(q-1)}\left(\equiv p_{0}\right)$. Note that $\frac{d \tilde{P}_{P}}{d p}=0$ also for $p=\frac{1}{\lambda}$ but for this case $p \geq 1$ and as a result is not considered.

It is required that $0 \leq p_{0} \leq 1 . p_{0} \geq 0$ is satisfied if $q-1-\lambda \overline{|S|} \geq 0$ or $\lambda \leq \frac{q-1}{\overline{|S|}} \cdot p_{0} \leq 1$ is satisfied if $\frac{q-1-\lambda \overline{|S|}}{\lambda(\overline{|S|}+1)(q-1)} \leq 1$

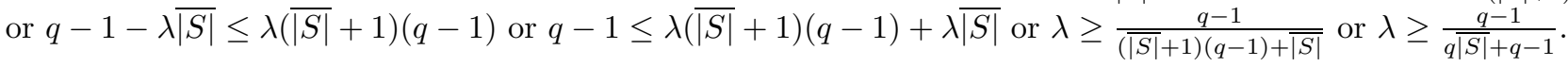




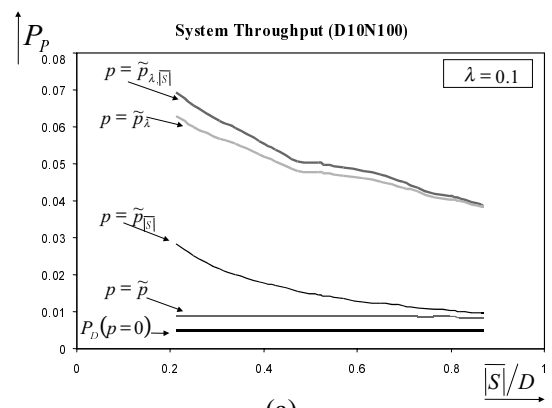

(a)

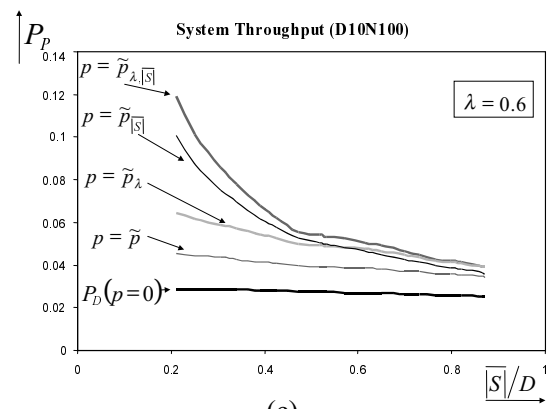

(c)

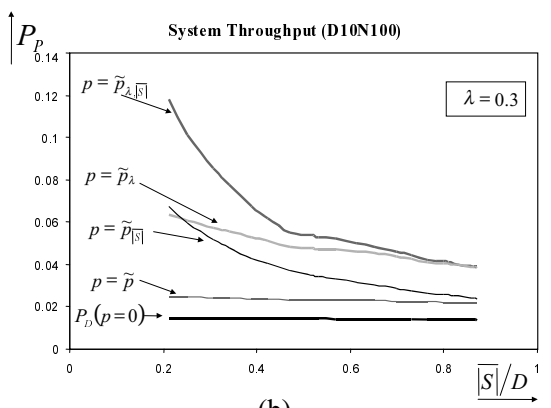

(b)

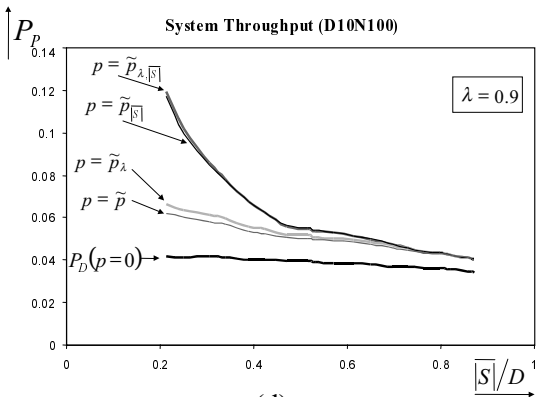

(d)

Fig. 13. System throughput simulation results for the D10N100 topology as a function the topology density $\overline{|S|} / D$ for different values of $p\left(p=0, p=\tilde{p}_{\lambda, \overline{S \mid}}, p=\tilde{p}_{\lambda}, p=\tilde{p}_{\overline{|S|}}\right.$ and $\left.p=\tilde{p}\right)$ and different values of $\lambda(\lambda=0.1, \lambda=0.3, \lambda=0.6$ and $\lambda=0.9)$.

\section{Accuracy of $1-\frac{1-\lambda}{1-p \lambda}$}

It is required that $\frac{1-\lambda}{1-p \lambda}$ is close to 1 or $1-\frac{1-\lambda}{1-p \lambda}$ close to 0 or $\lambda \frac{1-p}{1-p \lambda}$ close to 0 . Let $g(\lambda, p)=\lambda \frac{1-p}{1-p \lambda}$ and the closer $g(\lambda, p)$ to 0 the better the approximation. Obviously, $g(0, p)=g(\lambda, 1)=0$

It can be calculated that $\frac{d g(\lambda, p)}{d \lambda}=\frac{1-p}{(1-p \lambda)^{2}} \geq 0$. Consequently, as $\lambda$ increases $g(\lambda, p)$ increases. The maximum is assumed for $\lambda=1, g(1, p)=1$. Consequently, the smaller the value of $\lambda$ the better the approximation.

It can also be calculated that $\frac{d g(\lambda, p)}{d p}=-\lambda \frac{1-\lambda}{(1-p \lambda)^{2}} \leq 0$. Consequently, as $p$ increases $g(\lambda, p)$ decreases. The maximum is assumed for $p=0, g(\lambda, 0)=\lambda$. Consequently, the higher the value of $p$ the better the approximation.

\section{Accuracy of $\left|\hat{P}_{P}-\tilde{P}_{P}\right|$}

According to Equation (9) and Equation (10), $\left|\hat{P}_{P}-\tilde{P}_{P}\right|$ is calculated as follows.

$$
\begin{aligned}
\left|\hat{P}_{P}-\tilde{P}_{P}\right| & =\mid \frac{1}{N} \sum_{\forall u \in V} \frac{1+p(q-1)}{q} \lambda(1-p \lambda)^{\left|S_{v}\right|}-\frac{1+p(q-1)}{q} \lambda(1-p \lambda)^{\overline{|S|} \mid} \\
& =\frac{1+p(q-1)}{q} \lambda\left|\frac{1}{N} \sum_{\forall u \in V}(1-p \lambda)^{\left|S_{v}\right|}-(1-p \lambda)^{\overline{|S|}}\right| \\
& =\frac{1+p(q-1)}{q} \lambda(1-p \lambda)^{\overline{|S|}}\left|\frac{1}{N} \sum_{\forall u \in V}(1-p \lambda)^{\left|S_{v}\right|-\overline{|S|}}-1\right| .
\end{aligned}
$$

\section{Complementary of Theorem 4}

For $\frac{q-1}{q D+q-1} \leq \lambda<\frac{q-1}{q \overline{|S|}+q-1}, \frac{q-1-\lambda D}{\lambda(D+1)(q-1)} \leq 1$ will be shown that is satisfied. $\frac{q-1-\lambda D}{\lambda(D+1)(q-1)}$ can be written as $\frac{q-1}{(D+1)(q-1)}$. $\frac{\frac{q-1}{\lambda}-D}{(D+1)(q-1)}$ assumes the maximum value when the value for $\lambda$ is the minimum or $\lambda=\frac{q-1}{q D+q-1}$. Consequently, $\frac{\frac{q-1}{\lambda}-D}{(D+1)(q-1)} \leq$ $\frac{\frac{q-1}{q-1}-D}{\frac{q D+q-1}{(D+1)(q-1)}}=\frac{q D+q-1-D}{(D+1)(q-1)}=\frac{D(q-1)+q-1}{(D+1)(q-1)}=1$.

In order to show that $\frac{q-1-\lambda D}{\lambda(D+1)(q-1)} \leq \frac{q-1-\lambda \overline{|S|}}{\lambda(\overline{|S|}+1)(q-1)}$ it is enough to show that $\frac{q-1-\lambda D}{D+1} \leq \frac{q-1-\lambda \overline{|S|}}{\overline{|S|}+1}$ or $(\overline{|S|}+1)(q-1-\lambda D) \leq$ $(D+1)(q-1-\lambda \overline{|S|})$ or $\overline{|S|} q+q-\overline{|S|}-1-\lambda D \overline{|S|}-\lambda D \leq q D+q-D-1-\lambda \overline{|S|} D-\lambda \overline{|S|}$ or $\overline{|S|} q-\overline{|S|}-\lambda D \leq q D-D-\lambda \overline{|S|}$ or $\overline{|S|}(q-1+\lambda) \leq D(q-1+\lambda)$ or $\overline{|S|} \leq D$. The latter is always satisfied. 


\section{$14 \tilde{p}_{\overline{|S|}} \operatorname{Versus} \tilde{p}_{0_{\max }}$ and $\tilde{p}_{0_{\text {min }}}$}

In order to show that $\tilde{p}_{\overline{|S|}}<\tilde{p}_{0_{\text {max }}}$, it is enough to show that $\frac{q-1-\overline{|S|}}{(\overline{|S|}+1)(q-1)}<\frac{1}{\overline{|S|}+1}$ or $\frac{q-1-\overline{S \mid}}{q-1}<1$ or $q-1-\overline{|S|}<q-1$ or $\overline{|S|}>0$. The latter is always satisfied.

In order to show that $\tilde{p}_{\overline{|S|}}>\tilde{p}_{0_{\text {min }}}$, it is enough to show that $\frac{q-1-\overline{|S|}}{(\overline{|S|}+1)(q-1)}>\frac{q^{2}-(2 \overline{S \mid}+1)\left(q-\frac{2 \overline{S \mid}+1}{4}\right)}{\left(q^{2}-(\overline{|S|}+1)\left(q-\frac{2 \sqrt{S \mid}+1}{4}\right)\right)(\overline{|S|}+1)}$ or $\frac{q-1-\overline{|S|}}{q-1}>$ $\frac{q^{2}-(2 \overline{S \mid}+1)\left(q-\frac{2 \sqrt{S \mid}+1}{4}\right)}{q^{2}-(\overline{|S|}+1)\left(q-\frac{2 \overline{S \mid}+1}{4}\right)}$ or $\frac{q-1-\overline{|S|}}{q-1}>\frac{q^{2}-(\overline{|S|}+1)\left(q-\frac{2 \overline{S \mid}+1}{4}\right)-\overline{|S|}\left(q-\frac{2 \overline{S \mid}+1}{4}\right)}{q^{2}-(\overline{|S|}+1)\left(q-\frac{2 \bar{S} \mid+1}{4}\right)}$ or $1-\frac{\overline{|S|}}{q-1}>1-\frac{\overline{|S|}\left(q-\frac{2 \overline{S \mid}+1}{4}\right)}{q^{2}-(\overline{|S|}+1)\left(q-\frac{2 \bar{S} \mid+1}{4}\right)}$ or $\frac{1}{q-1}<$ $\frac{q-\frac{2 \sqrt{|S|}+1}{4}}{q^{2}-(\overline{|S|}+1)\left(q-\frac{2 \sqrt{S}+1}{4}\right)}$ or $q^{2}-(\overline{|S|}+1)\left(q-\frac{2 \overline{S S \mid}+1}{4}\right)<(q-1)\left(q-\frac{2 \overline{S \mid}+1}{4}\right)$ or $q^{2}<(q+\overline{|S|})\left(q-\frac{2 \overline{|S|}+1}{4}\right)$ or $4 q^{2}<$ $(q+\overline{|S|})(4 q-2 \overline{|S|}-1)$ or $4 q^{2}<4 q^{2}-2 q \overline{|S|}-q+4 q \overline{|S|}-2 \overline{|S|}^{2}-\overline{|S|}$ or $0<-q+2 q \overline{|S|}-2 \overline{|S|}^{2}-\overline{|S|}$ or $q-2 q|\overline{S \mid}+2| \overline{S \mid}^{2}+\overline{|S|}<0$ or $q(1-2 \overline{|S|})<-2 \overline{|S|}^{2}-\overline{|S|}$. Given that $1-2 \mid \overline{S \mid}<0$, it is enough that $q>\overline{|S|} \frac{2 \overline{S \mid}+1}{2 \overline{|S|}-1}$ is satisfied. Consequently, $\tilde{p}_{\overline{|S|}}>\tilde{p}_{0_{\text {min }}}$,

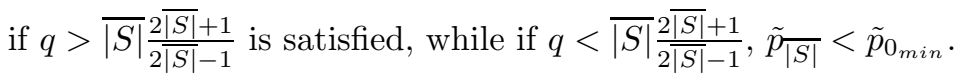

